\title{
Effects of Gc-Macrophage Activating Factor in Human Neurons; Implications for Treatment of Chronic Fatigue Syndrome
}

\author{
${ }^{1}$ Rodney Smith, ${ }^{1}$ Lynda Thyer, ${ }^{1}$ Emma Ward, ${ }^{2}$ Elisabetta Meacci, ${ }^{3}$ Jacopo J.V. Branca, \\ ${ }^{3}$ Gabriele Morucci, ${ }^{3}$ Massimo Gulisano, ${ }^{2}$ Marco Ruggiero, ${ }^{3}$ Alessandra Pacini, \\ ${ }^{3}$ Ferdinando Paternostro, ${ }^{4}$ Di Cesare Mannelli Lorenzo, ${ }^{5}$ David J. Noakes and ${ }^{3}$ Stefania Pacini \\ ${ }^{1}$ Macro Innovations Ltd, Cambridge, UK \\ ${ }^{2}$ Department of Experimental and Clinical Biomedical Sciences, University of Firenze, Firenze, Italy \\ ${ }^{3}$ Department of Experimental and Clinical Medicine, University of Firenze, L.go Brambilla, 3, 50134 Firenze, Italy \\ ${ }^{4}$ Department of Neuroscience, Psychology, Drug Research and Child Health, University of Firenze, Firenze, Italy \\ ${ }^{5}$ Immuno Biotech Ltd; Channel Isles, Guernsey
}

Received 2013-10-29, Revised 2013-11-05; Accepted 2013-11-06

\begin{abstract}
Myalgic Encephalomyelitis/Chronic Fatigue Syndrome (ME/CFS) is a debilitating disease of multifactorial aetiology characterized by immune system dysfunction, widespread inflammation, multisystemic neuropathology and persistent pain. Given the central role of the immune system in the pathogenesis of the syndrome, we studied the effects of a potent modulator of the immune system in in vitro and in vivo models that could help clarifying its role and indications in ME/CFS treatment. To this end, we studied the effects of vitamin D-binding protein-derived macrophage activating factor (also designated as GcMacrophage Activating Factor or (GcMAF)) on human neuronal cells (SH-SY5Y) and on the persistent pain induced by osteoarticular damage in rats. GcMAF at pM concentration increased neuronal cell viability and metabolism through increased mitochondrial enzyme activity. These effects were accompanied by cAMP formation and by morphological changes that were representative of neuronal differentiation. We hypothesize that these effects are to be ascribed to the interconnection between the GcMAF and Vitamin D Receptor (VDR) signalling pathways. The results presented here confirm at the experimental level the therapeutic effects of GcMAF in ME/CFS and elucidate the mechanisms of action through which GcMAF might be responsible for such therapeutic effects.
\end{abstract}

Keywords: Chronic Fatigue Syndrome, Immunotherapy, Vitamin D, Macrophage Activating Factor, GcMAF

\section{INTRODUCTION}

Myalgic Encephalomyelitis/Chronic Fatigue Syndrome (ME/CFS) is a complex disorder characterized by immune system dysfunction, widespread inflammation and multisystemic neuropathology (Carruthers et al., 2011; De Meirleir et al., 2013). Dysfunction of the immune system involves abnormal functions and distributions of $\mathrm{T}$ lymphocytes, $\mathrm{B}$ lymphocytes, natural killer cells and monocyte/macrophages (Uchida, 1992; Bansal et al., 2012). The aetiology of ME/CFS has still to be clearly defined and multiple factors may be responsible for its onset and progression, thus lending credit to the hypothesis that both aetiology and pathogenesis are multifactorial (Carruthers et al., 2011). Heavy metal exposure and viral infections are among the factors that contribute to ME/CFS aetiology and pathogenesis and a role for human endogenous retroviruses has been recently hypothesized (De Meirleir et al., 2013). Both

Corresponding Author: Stefania Pacini, Department of Experimental and Clinical Medicine, University of Firenze, L.go Brambilla, 3, 50134 Firenze, Italy Tel: (+39) 0554271810 
chronic heavy metal exposure and viral infections are considered responsible for the immune system dysfunction and neuropathology that are typical of ME/CFS (Pacini et al., 2012a; De Meirleir et al., 2013).

Among the cells of the immune system that play a role in the pathogenesis of ME/CFS, macrophages are considered of primary importance since the nervous and immune systems mutually cooperate via release of mediators of both neurological and immunological derivation (Covelli et al., 2005). Macrophages, originating from the migration and differentiation of circulating monocytes into virtually all tissues, are extremely flexible and plastic cells. They play vital homeostatic roles both in the nervous and immune systems, i.e., in those systems that are altered in ME/CFS (Castagna et al., 2012).

One of the most prominent regulators of macrophage function is the vitamin D-binding protein-derived macrophage activating factor, also designated as Gcmacrophage activating factor or GcMAF (Nagasawa et al., 2005). Macrophage activation by GcMAF induces a significant variation of surface receptors that in turn recognize abnormality in malignant cell surface and induce the apoptosis of cancerous cells as well as virus-infected cells (Yamamoto et al., 2009; Thyer et al., 2013a).

The interest for clinical use of GcMAF in a variety of conditions derives from the observation that different diseases involving the immune system such as cancer, viral infections and autoimmune diseases, show elevated level of serum alpha-N-acetylgalactosaminidase (Nagalase) an enzyme that deglycosylates vitamin Dbinding protein. This results in the loss of GcMAF precursor activity and consequent dysfunction of the immune system (Yamamoto et al., 2008a; 1997). Thus, serum Nagalase activity has been used as diagnostic indicator for a variety of conditions ranging from cancer to autism (Greco et al., 2009; Bradstreet et al., 2012). In this latter study it was demonstrated that GcMAF treatment of autistic children with elevated levels of Nagalase was associated with a significant improvement of autism symptoms, thus further stressing the interconnection between the immune and the nervous system.

It should be noticed, however, that GcMAF, in addition to stimulating macrophages, shows a number of other biological properties that contribute to its therapeutic effects. These range from inhibition of angiogenesis (Pacini et al., 2011) to direct inhibition of human cancer cell proliferation and metastatic potential (Gregory et al., 2010; Pacini et al., 2012b) to increased production of energy at the mitochondrial level (Pacini et al., 2013). We recently demonstrated that these multifaceted effects of GcMAF can be interpreted considering the interconnection between the GcMAF and the Vitamin D Receptor (VDR) signalling pathways (Thyer et al., 2013b).

Therefore, based on these premises, there is a solid scientific rationale to propose the use of GcMAF in $\mathrm{ME} / \mathrm{CFS}$, a syndrome characterized by alterations that can be targeted by this protein. In this study we describe in vitro and in vivo results that are consistent with a direct effect of GcMAF on human neuronal viability and metabolic activity and on inflammation-associated pain.

\section{MATERIALS AND METHODS}

\subsection{Reagents}

Commercially available GcMAF was obtained from Immuno Biotech Ltd, Guernsey, Channel Isles. GcMAF was purified according to the procedure previously described (Yamamoto et al., 2008b). Briefly, vitamin Dbinding protein (Gc-protein) was isolated from purified human serum obtained from the American Red Cross, using either 25-hydroxyvitamin D3-Sepharose high affinity chromatography or actin-agarose affinity chromatography. The bound material was eluted and then further processed by incubation with three immobilized enzymes as described (Bradstreet et al., 2012). The resulting GcMAF was filter sterilized. Protein content and concentration were assayed using standard Bradford protein assay methods (Bradford, 1976). Purity was assessed by SDS-PAGE and Western Blot analysis performed after each step of the preparation procedure; at the end of the procedure, only one band could be evidenced (Fig. 1A). At the end of the production process, GcMAF was checked for sterility in-house and externally by independent laboratories. Its safety and biological activity were tested in monocytes, human breast cancer cells and chick embryos (Pacini et al., 2011; 2012a; Thyer et al., 2013a). When not indicated otherwise, common reagents were from Sigma Aldrich, Milano, Italy.

\subsection{Cell Cultures}

Human SH-SY5Y neuronal cells were obtained from the Istituto Zooprofilattico Sperimentale della Lombardia e dell'Emilia-Romagna, Brescia, Italy. Cells were routinely maintained at $37^{\circ} \mathrm{C}$ in a humidified atmosphere of $5 \% \mathrm{CO} 2$ in Eagle's minimum essential medium in Earle's Balanced salt solution (45\%), Ham's F12 medium (45\%), foetal calf serum $10 \%$. In the experiments described in Fig. 2 and 3 and Table 1, the cells were starved for $24 \mathrm{~h}$ prior to the experiment in serum-free medium and the experiments were conducted in serum-free medium. 


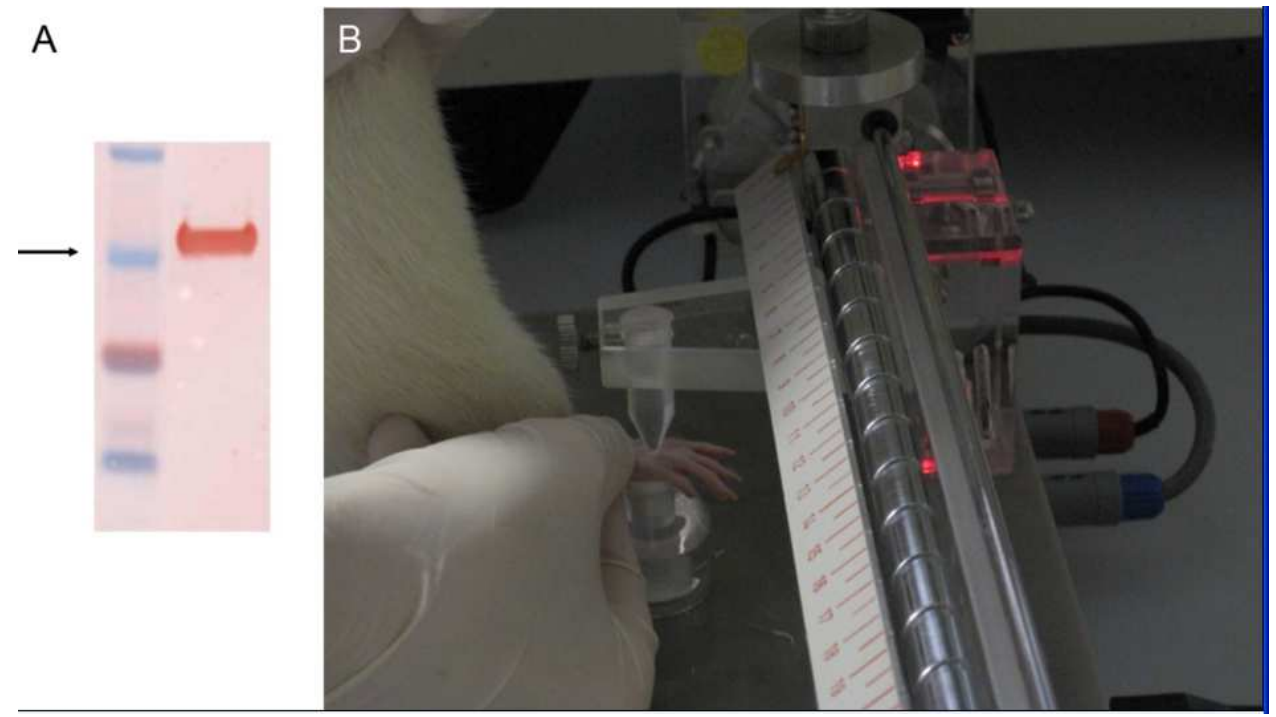

Fig. 1. (A) Western blot analysis of highly purified GcMAF. The presence of alpha- $N$-acetylgalactosamine was evidenced by blotting against biotin-labelled peanut lectin. Streptavidin labelled with horseradish peroxidase (Vector Labs, UK) was applied to the membrane and then stained with AEC (3-Amino-9-ethylcarbazole) staining kit (Sigma-Aldrich, UK). 15 $\mu \mathrm{L}$ of $100 \mu \mathrm{g} \mathrm{mL}^{-1} \mathrm{GcMAF}$ by total protein assay were applied and the electrophoresis was performed in MES running buffer. The molecular weight markers are SeeBlue ${ }^{\circledR}$ Plus2 Pre-Stained Standard (Invitrogen Life Technologies, UK). Left lane: molecular weight markers. Arrow indicates glutamic dehydrogenase standard. Right lane: GcMAF. (B) The analgesimeter to determine pain threshold. A constantly increasing pressure was applied to a small area of the dorsal surface of the paw using a blunt conical probe. Pressure was increased until a vocalization or a withdrawal reflex occurred. The picture was taken before beginning to apply the pressure and the circular weight is on the far left of the scale that is at the top of the picture

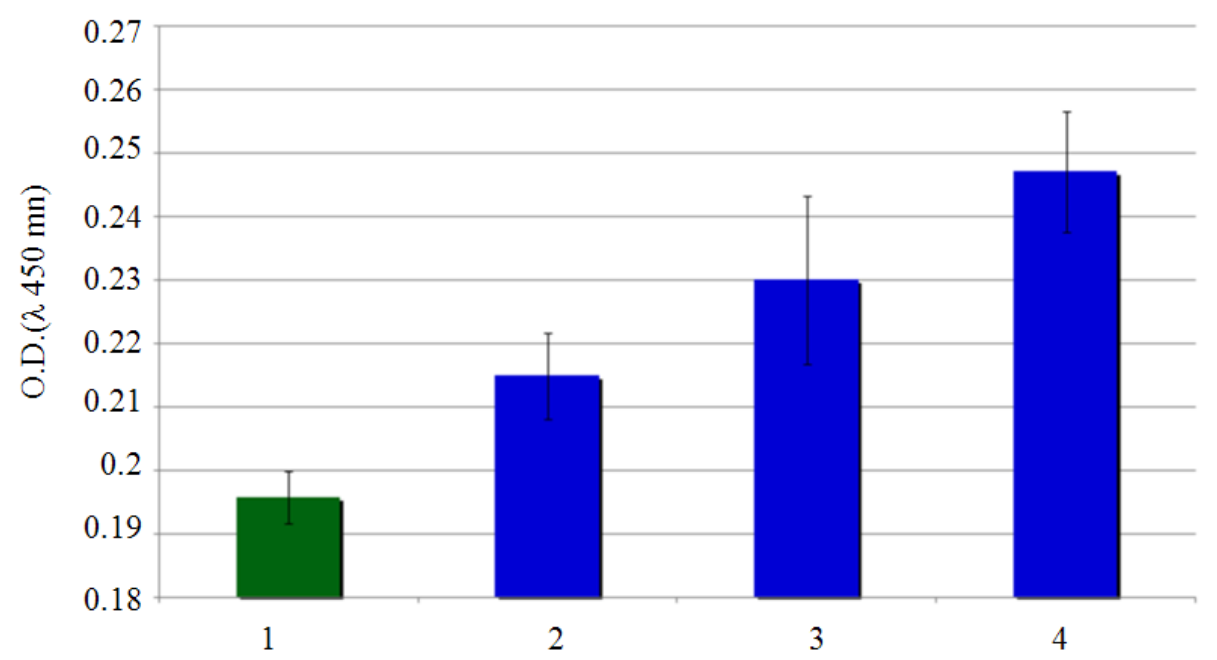

Fig. 2. Effects of GcMAF on human neuronal cell viability and metabolism. Human SH-SY5Y neuronal cell viability and metabolism were determined as described in Materials and Methods. GcMAF (8-800 pM) was added to cell cultures for $24 \mathrm{~h}$ in serum-free medium. Column 1: control (saline containing 800 pM Gc-protein). Columns 2, 3, 4: GcMAF 8, 80, 800 pM. Results were expressed as optical density at $450 \mathrm{~nm}$ wavelength and are means \pm S.E.M $(\mathrm{n}=12)$. All the data referring to GcMAF stimulation were statistically different from control $(\mathrm{p}<0.02)$ 

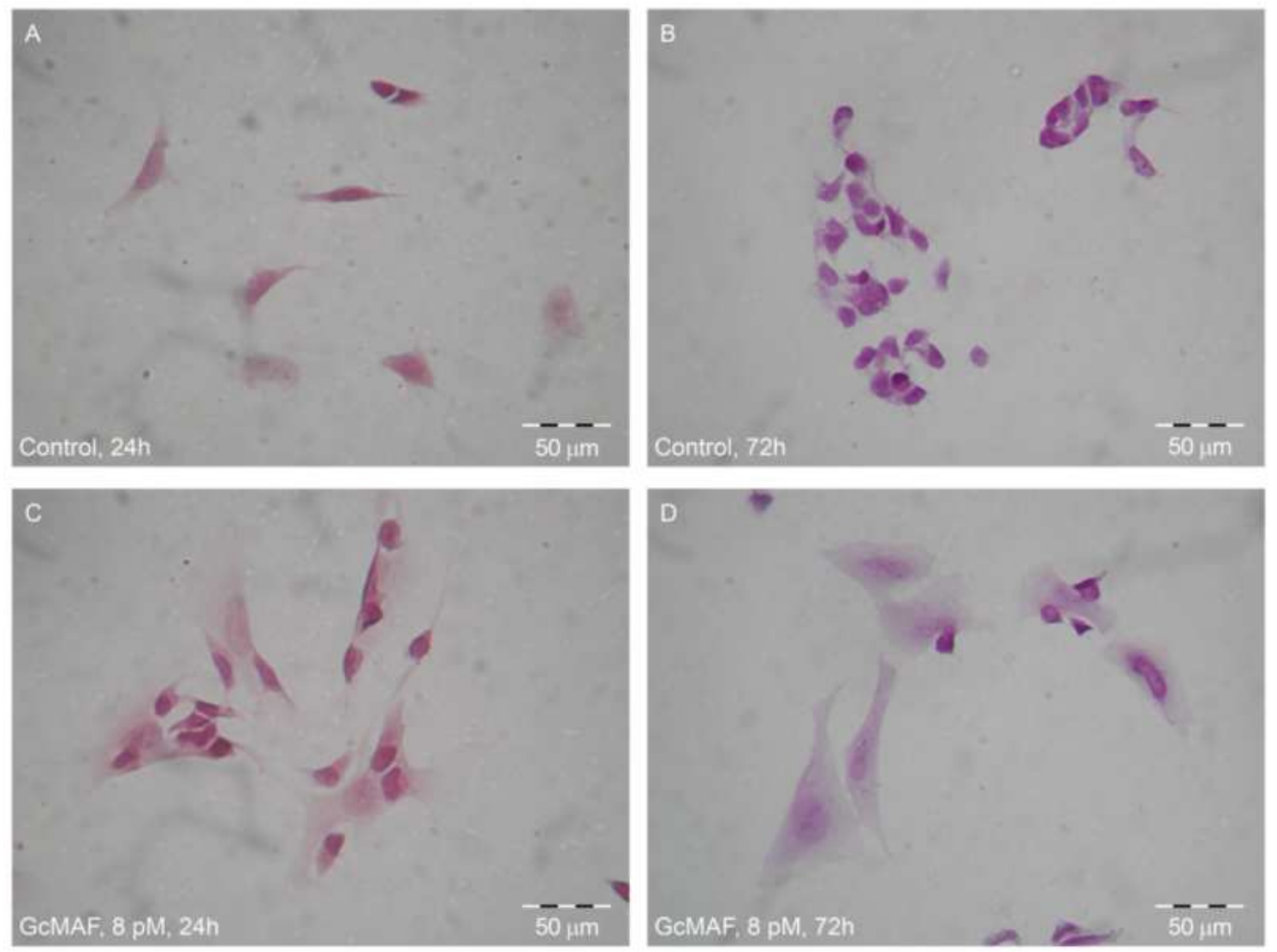

Fig. 3. Effects of GcMAF on human neuronal morphology. Human SH-SY5Y neuronal cells were stained with haematoxylin-eosin after $24 \mathrm{~h}$ or $72 \mathrm{~h}$ incubation with saline containing $8 \mathrm{pM}$ Gc-protein (control), or with $8 \mathrm{pM} \mathrm{GcMAF}$. A: control, $24 \mathrm{~h}$. B: control, 72 h. C: GcMAF, 8 pM, 24 h. D: GcMAF, 8 pM, 72 h

Table 1. cAMP concentration in human neuronal cells stimulated with GcMAF

\begin{tabular}{ll}
\hline Treatment & cAMP \\
\hline Control & $10.5 \pm 1.2$ \\
GcMAF $8 M$ & $12.0 \pm 1.1$ \\
GcMAF $80 \mathrm{pM}$ & $19.8 \pm 1.2^{*}$ \\
\hline
\end{tabular}

\subsection{Study of Cell Viability}

Assessment of cell viability and metabolic activity were determined by Cell Counting Kit-8 for quantitation of viable cell number (Sigma Aldrich). According to the Manufacturer, this assay measures active mitochondrial dehydrogenases of living cells. Each condition was replicated with quadruplicate samples and each experiment was replicated three times. Results are expressed as optical density (absorbance) at $450 \mathrm{~nm}$. Absorbance is directly proportional to cell viability and metabolic activity. Differences between experimental values were evaluated by the Student's t-test. In order to avoid biases, the experiments were performed in blind and the experimenters were not aware of the treatment.

\section{4. cAMP Assay}

3'-5'-cyclic Adenosine Monophosphate (cAMP) levels were measured by "cAMP Direct Immunoassay Kit" obtained from Abnova, Heidelberg, Germany. The kit utilizes recombinant Protein G coated 96-well plate to efficiently anchor cAMP polyclonal antibody onto the plate. CAMP-HRP conjugate directly competes with cAMP from sample binding to the cAMP antibody on the plate. After incubation and washing, the amount of cAMP-HRP bound to the plate can easily be determined by reading HRP activity at O.D. $450 \mathrm{~nm}$. The intensity of O.D. $450 \mathrm{~nm}$ is inversely proportional to the cAMP concentration in samples expressed as nM. Each condition was replicated in quadruplicate samples 
and each experiment was replicated three times. Differences between experimental points were evaluated by the Student's t-test.

\subsection{Studies on Animals}

Male Sprague-Dawley rats (Harlan, Varese, Italy) weighing approximately 200-250 $\mathrm{g}$ at the beginning of the experimental procedure were used for the experiments. The Institutional Animal Care and Use Committee (IACUC) or ethics committee that approved this study is the structure of the University of Firenze where the experiments with animals where performed that is the Centro Stabulazione Animali di Laboratorio (Ce.S.A.L., Centre for Stabulation of Laboratory Animals), Viale Pieraccini 650139 Firenze. Tel. +39.055.4271308. Fax. +39.055.4271203. e-mail: cesal@unifi.it. The Centre complies with all the E.U. laws concerning experiments on animals. Four animals per cage were housed at $23 \pm 1^{\circ} \mathrm{C}$ under a $12 \mathrm{~h}$ light/dark cycle; they were fed with standard laboratory diet and tap water ad libitum and used at least one week after their arrival. The experimental protocol complied with the European Community guidelines for animal care (DL 116/92, the European Communities Council Directive of 24 November 1986: 86/609/EEC). The ethical policy of the University of Firenze complies with the Guide for the Care and Use of Laboratory Animals of the US National Institutes of Health (NIH Publication no. 85-23, revised 1996; University of Firenze assurance number: A5278$01)$. Animals were anesthetized with $2 \%$ isoflurane before the surgical procedures and sacrifice, which was performed by cervical dislocation. All efforts were made to minimize suffering and reduce the number of animals used. Rats were randomly assigned to each experimental group and individually habituated to handling before testing. Unilateral osteoarthritis was induced by injection of Monoiodoacetate (MIA, Sigma Aldrich) into the tibiotarsal joint. On day 0 , rats were slightly anesthetized by $2 \%$ isoflurane, the left leg skin was sterilized with $75 \%$ ethyl alcohol and the lateral malleolus located by palpation; then, a 28-gauge needle was inserted vertically to penetrate the skin and turned distally for insertion into the articular cavity at the gap between the tibiofibular and tarsal bone until a distinct loss of resistance was felt. MIA dose of $2 \mathrm{mg}$ in $25 \mu \mathrm{L}$ saline was delivered into the left articular cavity (Mannelli et al., 2013). The paw pressure tests (see below) were performed at day 14 . GcMAF (25 ng) was i.p. administered. Control rats received $25 \mu \mathrm{L}$ of saline solution (day 0 ) in the tibiotarsal joint and saline containing $25 \mathrm{ng}$ of Gc-protein i.p. at day 14. The pain threshold in the rat was determined with an analgesimeter (Ugo Basile, Varese, Italy) as described (Mannelli et al., 2013). Briefly, a constantly increasing pressure was applied to a small area of the dorsal surface of the paw using a blunt conical probe (Fig. 1B). Pressure was increased until a vocalization or a withdrawal reflex occurred. The withdrawal threshold was expressed in grams, the test was repeated twice and the mean was considered as the value for each paw. In order to avoid biases, the experiments were performed in a blinded manner where the experimenters were not aware of the treatment. It was ascertained that recorded pressure values did not vary when repetitively measured during the experimental session.

\section{RESULTS}

\subsection{Effects of GcMAF on Human Neurons}

It is well established that significant neuroanatomical changes occur in ME/CFS and they are consistent with the neuropathologic symptoms that are characteristic of this syndrome. As recently demonstrated, the most evident neuroanatomical alteration in the brain of $\mathrm{ME} / \mathrm{CFS}$ patients is the reduction of the grey and white matter volume in several anatomical areas of the brain (Puri et al., 2012). It can be hypothesized that reduction of grey and white matter volume corresponds to decreased connectivity between neurons with consequent impairment of function as it has been observed in autism (Zikopoulos et al., 2013). Therefore, it can be proposed that factors stimulating neuronal viability and metabolism might counteract such a reduction of grey matter volume and connectivity thereby improving clinical symptoms in ME/CFS patients. Having noticed a significant improvement of neurological symptoms in ME/CFS patients (see below), we decided to study the effects of GcMAF on human neurons using a well established in vitro experimental system. To this end we used human SH-SY5Y neuronal cells, a cellular in vitro model considered useful in many areas of neuroscience research (Agholme et al., 2010). In fact, these cells represent a model system to study the neurobiology of neurodegenerative diseases (Xie et al., 2010) and they are used to evaluate the neuroprotective effects of a variety of substances (Thapa et al., 2013). In addition, these cells express the VDR receptor, thus making them suitable to study those effects of GcMAF that 
are interconnected with VDR receptor signalling (Celli et al., 1999; Thyer et al., 2013b). Figure 2, shows that GcMAF $(8-800$ pM) significantly increased neuronal cell viability and metabolic activity in a dose-dependent manner, with significant effects observed even at the lowest $\mathrm{pM}$ concentration. These effects on cell viability were associated with dosedependent intracellular cAMP production (Table 1). The results shown in Fig. 2 and Table 1 as well as those presented in Fig. $\mathbf{3}$ were obtained in serum-free medium, thus ruling out the effects of possible confounding factors present in serum (Inui et al., 2013).

Increased viability and metabolic activity following GcMAF stimulation as well as cAMP formation were consistent with the morphological changes induced by GcMAF in human neurons and shown in Fig. 3. In the absence of GcMAF, SH-SY5Y neuronal cells, stained with haematoxylin-eosin, appeared as small, relatively undifferentiated cells with large nuclei (Fig. 3A and 3B). After $24 \mathrm{~h}$ stimulation with 8 pM GcMAF, several cells showed a significant change in morphology that was consistent with the induction of neuronal differentiation and increased connectivity (Fig. 3C). The cytoplasm was enlarged and several cytoplasmic elongations could be observed. After $72 \mathrm{~h}$ incubation with 8 pM GcMAF, these morphological changes were more evident and well differentiated cells could be observed (Fig. 3D). After incubation with GcMAF, the cells appeared to establish contacts with each other. Taken together these results indicate that GcMAF at $\mathrm{pM}$ concentration increases neuronal cell viability, metabolic activity and differentiation, with the first effects being observed at 24 h. Considering the well demonstrated role of cAMP in the induction of SH-SY5Y differentiation (Kume et al., 2008), it can be hypothesized that these effects of GcMAF are mediated by the cAMP signalling pathway involving the Extracellular signal-Regulated protein Kinase (ERK) and the nuclear factor kappa B (NF- $\kappa \mathrm{B})$ pathways (Sun et al., 2012). As it was recently demonstrated, this latter signalling pathway is strictly interconnected with the VDR signalling pathway in human mononuclear cells (Nakou et al., 2010), thus lending credit to the hypothesis that the effects of GcMAF are due to a complex, interconnected, network of intracellular signalling. It is worth noticing that the assay that we used to determine cell viability measures mitochondrial activity and mitochondrial dysfunction is known to be hallmark of ME/CFS (Booth et al., 2012). Therefore, the effects of GcMAF described here might directly counteract one of the basic alterations of $\mathrm{ME} / \mathrm{CFS}$ at the sub-cellular level.

\subsection{Effects of GcMAF on Inflammatory Pain}

There exist several animal models to study the neurobiology of fatigue and many of them are centered on immunologically induced fatigue (Harrington, 2012). However, it is well assessed that, in addition to fatigue, persistent pain and systemic inflammation are the most debilitating symptoms of ME/CFS. Because of this consideration, we decided to evaluate the effects of GcMAF on an experimental model of persistent pain instead of using a model of fatigue.

In fact, it has been hypothesized that mitochondrial dysfunction is involved in muscle pain and central sensitization is typically observed in these patients (Meeus et al., 2013). Therefore, having observed a significant stimulatory effects of GcMAF on mitochondrial activity in human neurons (Fig. 2), we decided to study its effects in a well-established model of inflammatory pain that is the osteoarthritic pain induced by MIA (Mannelli et al., 2013). It is well assessed that morphological alterations are associated with a persistent inflammatory pain which, starting from the 14th day after MIA injection, possesses a neuropathic component (Ivanavicius et al., 2007). Nonsteroidal antiinflammatory drugs such as diclofenac can reduce MIAdependent pain during the first inflammatory phase, but they are ineffective in the degenerative neuropathic phase (Fernihough et al., 2004), while gabapentin, an antiepileptic molecule widely used to treat neuropathic pain in adult patients (Davis and Srivastava, 2003), is effective (Ivanavicius et al., 2007).

The effectiveness of GcMAF was evaluated after acute i.p. administration ( $25 \mathrm{ng}, 15 \mathrm{~min}$ before the test). As expected, fourteen days after MIA, the weight tolerated on the ipsilateral paw (MIA), was significantly reduced as compared to the contralateral paw and control animals. The weight tolerated on the ipsilateral paw was $30 \pm 4 \mathrm{~g}$, whereas the weight tolerated on the contralateral healthy paw and in control animals was $60 \pm 5 \mathrm{~g}$. GcMAF, 15 min after i.p. administration, increased the withdrawal threshold and it was still effective after $24 \mathrm{~h}$. Thus, the weight tolerated on the ipsilateral paw was $55 \pm 6 \mathrm{~g}$, that is almost identical to the weight tolerated on the contralateral paw and in control animals. It is worth noticing that GcMAF did not modify the weight tolerated in the contralateral healthy paw, that remained $60 \pm 5 \mathrm{~g}$. This demonstrates that the effects of GcMAF were not to be attributed to non-specific 
analgesia. In analogy with the results presented in (Ivanavicius et al., 2007), it can be concluded that GcMAF counteracts the degenerative neuropathic phase that is responsible for the neuropathic pain.

\section{DISCUSSION}

ME/CFS is a debilitating disease of multifactorial aetiology with infectious as well as non-infectious factors possibly involved. In fact, it has been observed that symptoms often follow a viral infection or a period of stress even though a causal relationship with any particular type of infectious agent has yet to be defined (Bansal et al., 2012). If the aetiology is still ambiguous, the pathogenetic mechanisms, however, appear to be more defined and include immune system dysfunction as well as anatomical changes in certain areas of the brain that control the neurological functions impaired in ME/CFS patients (Puri et al., 2012).

GcMAF is a protein that controls a number of events at the molecular and cellular level resulting in a variety of biological effects that have been exploited to treat several clinical conditions, ranging from cancer to autism (Yamamoto et al., 2008c; Bradstreet et al., 2012). The rationale for using GcMAF in such diverse clinical conditions stems from the observation that in those conditions endogenous production of GcMAF is impaired by elevated serum levels of Nagalase. Nagalase is an enzyme whose activity is elevated in the serum of cancer patients as well as in the serum of patients with viral infections or autoimmune diseases (Yamamoto et al., 2009). Nagalase impairs endogenous production of GcMAF because it deglycosylates vitamin D-binding protein, the precursor of GcMAF, thus preventing the conversion of vitamin D-binding protein to active GcMAF (Yamamoto et al., 1997). Recent evidence, however, suggests that the therapeutic results observed with GcMAF are due not only to macrophage activation, but also to other direct effects of GcMAF on cell signalling and metabolism. We recently proposed that these effects may be explained considering the interconnection between GcMAF and VDR signalling pathways (Thyer et al., 2013b). The results presented in this study suggest a further interconnection with other signalling pathways that include ERK and NF-kB. This complex web of intracellular signalling could then be responsible for the diversified effects of GcMAF in different cell types and therefore, in different diseases.
In this study we demonstrate for the first time a direct effect of GcMAF on human neurons. In fact, GcMAF significantly improves human neuronal cell viability and metabolism and efficiently counteracts inflammatory/neuropathic pain in experimental animals. Considering that neurological symptoms, inflammation and persistent pain are the most debilitating features of $\mathrm{ME} / \mathrm{CFS}$, the results reported here explain the therapeutic effects reported by clinicians and patients using GcMAF in ME/CFS. In fact, several clinical observations on the efficacy of GcMAF in ME/CFS have been presented at international congresses. Among these, the results presented at the biennial meeting of the International Association for CFS and ME, held in Ottawa, Ontario, in 2011 (CFS, 2011) and those presented by researchers and patients at the GcMAF Immunology Conference, 2013 in Frankfurt, Germany. Consistent with these congress presentations, a very recent peer-reviewed paper reported the therapeutic effects of GcMAF on a variety of neurological diseases, including ME/CFS (Thyer et al., 2013a).

The results described in the present study suggest that the therapeutic efficacy of GcMAF in ME/CFS as well as in other cognate conditions such as autism (Bradstreet et al., 2012) in addition to immunomodulation, may be ascribed also to direct effects on neurons and neuropathic pain.

The results described in this study and the clinical observations quoted above raise two questions:

- Is GcMAF able to cross the blood brain barrier and act directly on central nervous system neurons increasing their metabolic activity and restoring connectivity?

- Which is the mechanism underlying the rapid effects of GcMAF on inflammatory pain?

Although we have no experimental data to answer the first question, it could be hypothesized that the interconnection between GcMAF and VDR signaling could favor transport through the blood brain barrier. In fact, transport of macromolecules across the blood brain barrier requires both specific and nonspecific interactions between macromolecules and proteins/receptors expressed on the luminal and/or the abluminal surfaces of the brain capillary endothelial cells (Xiao and Gan, 2013). Since VDR signaling appears to enhance brain to blood transport at the blood brain barrier through both genomic and nongenomic actions, it can be hypothesized that the 
interaction between GcMAF and VDR (Thyer et al., 2013a) may favor GcMAF transport into the brain.

The interconnection between GcMAF and VDR signaling pathways might also be responsible for the rapid effects of GcMAF on inflammatory neuropathic pain observed in rats with MIA-induced osteoarthritis. In fact, a recent report demonstrated that activation of VDR was associated with significant reduction of musculoskeletal pain associated with acute phase inflammatory response in women (Catalano et al., 2012).

\section{CONCLUSION}

The results presented here confirm at the experimental level the therapeutic effects of GcMAF in $\mathrm{ME} / \mathrm{CFS}$ and elucidate the molecular mechanisms of action through which GcMAF might be responsible for such therapeutic effects.

\section{ACKNOWLEDGMENT}

Marco Ruggiero, Stefania Pacini and Massimo Gulisano received grants from the University of Firenze and the Project PRIN 2009.

\subsection{Potential Conflicts of Interests}

David J. Noakes is the CEO of Immuno Biotech, Ltd (the company isolating and purifying the GcMAF protein). In order to avoid biases, the experiments were performed in blind and the experimenters, including DN, were not aware of the treatment.

Since November $1^{\text {st }}, 2013$, Marco Ruggiero is consulting scientific director of Immuno Biotech Ltd.

\section{REFERENCES}

Agholme, L., T. Lindstrom, K. Kagedal, J. Marcusson and M. Hallbeck, 2010. An in vitro model for neuroscience: Differentiation of SH-SY5Y cells into cells with morphological and biochemical characteristics of mature neurons. J. Alzheimer's Dis., 20: 1069-1082. DOI: 10.3233/JAD-2010-091363

Bansal, A.S., A.S. Bradley, K.N. Bishop, S. KianiAlikhan and B. Ford, 2012. Chronic fatigue syndrome, the immune system and viral infection. Brain Behav. Immunity, 26: 24-31. DOI: 10.1016/j.bbi.2011.06.016

Booth, N.E., S. Myhill and J. McLaren-Howard, 2012. Mitochondrial dysfunction and the pathophysiology of Myalgic Encephalomyelitis/Chronic Fatigue Syndrome (ME/CFS). Int. J. Clin. Exp. Med., 5: 208-220.
Bradford, M.M., 1976. A rapid and sensitive method for the quantitation of microgram quantities of protein utilizing the principle of protein-dye binding. Analytical Biochem., 72: 248-254. DOI: 10.1016/0003-2697(76)90527-3

Bradstreet, J.J., E. Vogelaar and L. Thyer, 2012. Initial observations of elevated alpha-nacetylgalactosaminidase activity associated with autism and observed reductions from gc proteinmacrophage activating factor injections. Autism Insights, 4: 31-38. DOI: 10.4137/AUI.S10485

Carruthers, B.M., M.I. van de Sande, K.L. De Meirleir, N.G. Klimas and G. Broderick et al., 2011. Myalgic encephalomyelitis: International consensus criteria. J. Internal Med., 270: 327-338. DOI: 10.1111/j.1365-2796.2011.02428.x

Castagna, A., R. Polati, A.M. Bossi and D. Girelli, 2012. Monocyte/macrophage proteomics: Recent findings and biomedical applications. Expert Rev. Proteom., 9: 201-215. DOI: 10.1586/epr.12.11

Catalano, A., N. Morabito, M. Atteritano, G. Basile and D. Cucinotta et al., 2012. Vitamin D reduces musculoskeletal pain after infusion of zoledronic acid for postmenopausal osteoporosis. Calcified Tissue Int., 90: 279-285. DOI: 10.1007/s00223-0129577-6

Celli, A., C. Treves, P. Nassi and M. Stio, 1999. Role of 1,25-dihydroxyvitamin D3 and extracellular calcium in the regulation of proliferation in cultured $\mathrm{SH}$ SY5Y human neuroblastoma cells. Neurochemical Res., 24: 691-698.

CFS, 2011. IACFS/ME international conference summary. Acceletare CFS Research.

Covelli, V., M.E. Passeri, D. Leogrande, E. Jirillo and L. Amati, 2005. Drug targets in stress-related disorders. Curr. Med. Chem., 12: 1801-1809. DOI: 10.2174/0929867054367202

Davis, M.P. and M. Srivastava, 2003. Demographics, assessment and management of pain in the elderly. Drugs Ag., 20: 23-57. DOI: 10.2165/00002512200320010-00003

De Meirleir, K.L., S.F. Khaiboullina, M. Fremont, J. Hulstaert and A.A. Rizvanov et al., 2013. Plasmacytoid dendritic cells in the duodenum of individuals diagnosed with myalgic encephalomyelitis are uniquely immunoreactive to antibodies to human endogenous retroviral proteins. Vivo, 27: 177-187. 
Fernihough, J., C. Gentry, M. Malcangio, A. Fox and J. Rediske et al., 2004. Pain related behaviour in two models of osteoarthritis in the rat knee. Pain, 112: 83-93. DOI: 10.1016/j.pain.2004.08.004

Greco, M., M.D. Mitri, F. Chiriacò, G. Leo and E. Brienza et al., 2009. Serum proteomic profile of cutaneous malignant melanoma and relation to cancer progression: Association to tumor derived alpha-N-acetylgalactosaminidase activity. Cancer Lett., 283: 222-229. DOI: 10.1016/j.canlet.2009.04.001

Gregory, K.J., B. Zhao, D.R. Bielenberg, S. Dridi and J. $\mathrm{Wu}$ et al., 2010. Vitamin $\mathrm{D}$ binding proteinmacrophage activating factor directly inhibits proliferation, migration and UPAR expression of prostate cancer cells. PLoS One, 5: e13428-e13428. DOI: 10.1371/journal.pone.0013428

Harrington, M.E., 2012. Neurobiological studies of fatigue. Progress Neurobiol., 99: 93-105. DOI: 10.1016/j.pneurobio.2012.07.004

Inui, T., D. Kuchiike, K. Kubo, M. Mette and Y. Uto et al., 2013. Clinical experience of integrative cancer immunotherapy with GcMAF. Anticancer Res., 33: 2917-2919.

Ivanavicius, S.P., A.D. Ball, C.G. Heapy, F.R. Westwood and F. Murray et al., 2007. Structural pathology in a rodent model of osteoarthritis is associated with neuropathic pain: Increased expression of ATF-3 and pharmacological characterisation. Pain, 128: 272-282. DOI: 10.1016/j.pain.2006.12.022

Kume, T., Y. Kawato, F. Osakada, Y. Izumi and H. Katsuki et al., 2008. Dibutyryl cyclic AMP induces differentiation of human neuroblastoma SH-SY5Y cells into a noradrenergic phenotype. Neurosci. Lett., 443: 199-203. DOI: 10.1016/j.neulet.2008.07.079

Mannelli, L.D.C., D. Bani, A. Bencini, M.L. Brandi and L. Calosi et al., 2013. Therapeutic effects of the superoxide dismutase mimetic compound $\mathrm{Mn}^{\mathrm{II}} \mathrm{Me}_{2} \mathrm{DO} 2 \mathrm{~A}$ on experimental articular pain in rats. Mediators Inflammat., 2013: 905360-905370. DOI: 10.1155/2013/905360

Meeus, M., J. Nijs, L. Hermans, D. Goubert and P. Calders, 2013. The role of mitochondrial dysfunctions due to oxidative and nitrosative stress in the chronic pain or chronic fatigue syndromes and fibromyalgia patients: Peripheral and central mechanisms as therapeutic targets? Expert Opin. Therapeutic Targets, 17: 1081-1089. DOI: $10.1517 / 14728222.2013 .818657$
Nagasawa, H., Y. Uto, H. Sasaki, N. Okamura and A. Murakami et al., 2005. Gc protein (vitamin Dbinding protein): Gc genotyping and GcMAF precursor activity. Anticancer Res., 25: 36893695.

Nakou, M., G. Bertsias, I. Stagakis, M. Centola and I. Tassiulas et al., 2010. Gene network analysis of bone marrow mononuclear cells reveals activation of multiple kinase pathways in human systemic lupus erythematosus. PLoS One, 5: e13351e13351. DOI: 10.1371/journal.pone.0013351

Pacini, S., G. Morucci, J.J.V. Branca, S. Aterini, M. Amato, et al. 2013. Effects of vitamin D3 and paricalcitol on immature cardiomyocytes: A novel role for vitamin $\mathrm{d}$ analogs in the prevention of cardiovascular diseases. Nutrients, 5: 2076-2092. DOI: 10.3390/nu5062076

Pacini, S., G. Morucci, T. Punzi, M. Gulisano and M. Ruggiero, 2011. Gc protein-derived MacrophageActivating Factor (GcMAF) stimulates cAMP formation in human mononuclear cells and inhibits angiogenesis in chick embryo chorionallantoic membrane assay. Cancer Immunol. Immunotherapy, 60: 479-485. DOI: 10.1007/s00262-010-0953-7

Pacini, S., M.G. Fiore, S. Magherini, G. Morucci and J.J. Branca et al., 2012a. Could cadmium be responsible for some of the neurological signs and symptoms of myalgic encephalomyelitis/chronic fatigue syndrome. Med. Hypotheses, 79: 403-407. DOI: 10.1016/j.mehy.2012.06.007

Pacini, S., T. Punzi, G. Morucci, M. Gulisano and M. Ruggiero, 2012b. Effects of vitamin D-binding protein-derived macrophage-activating factor on human breast cancer cells. Anticancer Res., 32: 45-52.

Puri, B.K., P.M. Jakeman, M. Agour, K.D. Gunatilake and K.A. Fernando et al., 2012. Regional grey and white matter volumetric changes in myalgic encephalomyelitis (chronic fatigue syndrome): A voxel-based morphometry $3 \mathrm{~T}$ MRI study. Br. J. Radiol., 85: e270-e273. DOI: 10.1259/bjr/93889091

Sun, Y.L., X.Y. Zhang, N. He, T. Sun and Y. Zhuang et al., 2012. Neuropeptide FF activates ERK and NF kappa B signal pathways in differentiated SH-SY5Y cells. Peptides, 38: 110-117. DOI: 10.1016/j.peptides.2012.08.019 
Thapa, A., B.C. Vernon, K. De la Peña, G. Soliz and H.A. Moreno et al., 2013. Membrane-mediated neuroprotection by curcumin from amyloid- $\beta$ peptide-induced toxicity. Langmuir, 29: 1171311723. DOI: 10.1021/la4020459

Thyer, L., E. Ward, R. Smith, J.J.V. Branca, G. Morucci et al., 2013b. Therapeutic effects of highly purified de-glycosylated GcMAF in the immunotherapy of patients with chronic diseases. Am. J. Immunol., 9: 78-84.

Thyer, L., E. Ward, R. Smith, M.G. Fiore and S. Magherini et al., 2013a. A novel role for a major component of the vitamin D Axis: Vitamin D binding protein-derived macrophage activating factor induces human breast cancer cell apoptosis through stimulation of macrophages. Nutrients, 5: 2577-2589. DOI: 10.3390/nu5072577

Uchida, A., 1992. Chronic fatigue immune dysfunction syndrome. Nihon Rinsho, 50: 2625-2629.

Xiao, G. and L.S. Gan, 2013. Receptor-mediated endocytosis and brain delivery of therapeutic biologics. Int. J. Cell Biol., 2013: 703545. DOI: $10.1155 / 2013 / 703545$

Xie, H.R., L.S. Hu and G.Y. Li, 2010. SH-SY5Y human neuroblastoma cell line: In vitro cell model of dopaminergic neurons in Parkinson's disease. Chinese Med. J., 123: 1086-1092. DOI: 10.3760/cma.j.issn.0366-6999

Yamamoto, N., H. Suyama and N. Yamamoto, 2008b. Immunotherapy for prostate cancer with gc protein-derived macrophage-activating factor, GcMAF. Translat. Oncol., 1: 65-72. DOI: 10.1593/tlo.08106
Yamamoto, N., H. Suyama, H. Nakazato, N. Yamamoto and Y. Koga, 2008c. Immunotherapy of metastatic colorectal cancer with vitamin D-binding proteinderived macrophage-activating factor, GcMAF. Cancer Immunol. Immunotherapy, 57: 1007-1016. DOI: $10.1007 / \mathrm{s} 00262-007-0431-\mathrm{Z}$

Yamamoto, N., H. Suyama, N. Yamamoto and N. Ushijima, 2008a. Immunotherapy of metastatic breast cancer patients with vitamin D-binding protein-derived Macrophage Activating Factor (GcMAF). Int. J. Cancer, 122: 461-467. DOI: $10.1002 /$ ijc. 23107

Yamamoto, N., N. Ushijima and Y. Koga, 2009. Immunotherapy of HIV-infected patients with Gc protein-derived Macrophage Activating Factor (GcMAF). J. Med. Virol., 81: 16-26. DOI: 10.1002/jmv.21376

Yamamoto, N., V.R. Naraparaju, M. Moore and L.H. Brent, 1997. Deglycosylation of serum vitamin D3binding protein by alpha-N-acetylgalactosaminidase detected in the plasma of patients with systemic lupus erythematosus. Clin. Immunol. Immunopathol., $\quad$ 82: 290-298. DOI: 10.1006/clin.1996.4320

Zikopoulos, B. and H. Barbas, 2013. Altered neural connectivity in excitatory and inhibitory cortical circuits in autism. Front Hum. Neurosci., 7: 609609. DOI: $10.3389 /$ fnhum.2013.00609 